\title{
Exploring the socio-economic and environmental components of infectious diseases using multivariate geovisualization: a case study of West Nile Virus
}

\author{
Abhishek K Kala ${ }^{\text {Equal first author, 1,2 }}$, Samuel F Atkinson ${ }^{\text {Corresp., Equal first author, 1, } 2 \text {, Chetan Tiwari }}{ }^{1,3}$ \\ ${ }^{1}$ Advanced Environmental Research Institute, University of North Texas, Denton, Texas, United States \\ 2 Department of Biological Sciences, University of North Texas, Denton, Texas, United States \\ 3 Department of Geography and the Environment, University of North Texas, Denton, Texas, United States \\ Corresponding Author: Samuel F Atkinson \\ Email address: atkinson@unt.edu
}

Background. This study postulates that underlying environmental conditions and a susceptible population's socio-economic status should be explored simultaneously to adequately understand a vector borne disease infection risk. Here we focus on West Nile Virus (WNV), a mosquito borne pathogen, as a case study for spatial data visualization of environmental characteristics of a vector's habitat alongside human demographic composition for understanding potential public health risks of infectious disease. Multiple efforts have attempted to predict WNV environmental risk, while others have documented factors related to human vulnerability to the disease. However, analytical modeling that combines the two is difficult due to the number of potential explanatory variables, varying spatial resolutions of available data, and differing research questions that drove the initial data collection. We propose that the use of geovisualization may provide a glimpse into the large number of potential variables influencing the disease and help distill them into a smaller number that might reveal hidden and unknown patterns. This geovisual look at the data might then guide development of analytical models that can combine environmental and socio-economic data.

Methods. Geovisualization was used to integrate an environmental model of the disease vector's habitat alongside human risk factors derived from socio-economic variables. County level WNV incidence rates from California, USA, were used to define aa geographically constrained study area where environmental and socio-economic data were extracted from 1,133 census tracts. A previously developed mosquito habitat model that was significantly related to WNV infected dead birds was used to describe the environmental components of the study area. Self-organizing maps found 49 clusters, each of which contained census tracts that were more similar to each other in terms of WNV environmental and socioeconomic data. Parallel coordinate plots permitted visualization of each cluster's data, uncovering patterns that allowed final census tract mapping exposing complex spatial patterns contained within the clusters.

Results. Our results suggest that simultaneously visualizing environmental and socio-economic data supports a fuller understanding of the underlying spatial processes for risks to vector-borne disease. Unexpected patterns were revealed in our study that would be useful for developing future multilevel analytical models. For example, when the cluster that contained census tracts with the highest median age was examined, it was determined that those census tracts only contained moderate mosquito habitat risk. Likewise, the cluster that contained census tracts with the highest mosquito habitat risk had populations with moderate median age. Finally, the cluster that contained census tracts with the highest WNV human incidence rates had unexpectedly low mosquito habitat risk.

Peer] reviewing PDF | (2020:02:46052:2:0:NEW 24 Jun 2020) 


\section{Exploring the socio-economic and environmental}

2 components of infectious diseasesid using

3 multivariate geovisualization: a case study of West

4 Nile Virus

${ }^{1}$ Advanced Environmental Research Institute, University of North Texas, Denton, TX, United States of America

${ }^{2}$ Department of Biological Sciences, University of North Texas, Denton, TX, United States of America

${ }^{3}$ Department of Geography and the Environment, University of North Texas, Denton, TX, United States of America

Corresponding Author:

Samuel F. Atkinson

Email address: atkinson@unt.edu

\section{Abstract}

Background. This study postulates that underlying environmental conditions and a susceptible population's socio-economic status should be explored simultaneously to adequately understand a vector borne disease infection risk. Here we focus on West Nile Virus (WNV), a mosquito borne pathogen, as a case study for spatial data visualization of environmental characteristics of a vector's habitat alongside human demographic composition for understanding potential public health risks of infectious disease. Multiple efforts have attempted to predict WNV environmental risk, while others have documented factors related to human vulnerability to the disease. However, analytical modeling that combines the two is difficult due to the number of potential explanatory variables, varying spatial resolutions of available data, and differing research questions that drove the initial data collection. We propose that the use of geovisualization may provide a glimpse into the large number of potential variables influencing the disease and help distill them into a smaller number that might reveal hidden and unknown patterns. This geovisual look at the data might then guide development of analytical models that can combine environmental and socio-economic data. Methods. Geovisualization was used to integrate an environmental model of the disease vector's habitat alongside human risk factors derived from socio-economic variables. County level WNV 
39

40

41

42

43

44

45

46

47

48

49

50

51

52

53

54

55

56

57

58

59

60

61

62

63

64

65

66

67

68

69

70

71

72

73

74

75

76

77

78

previously developed mosquito habitat model that was significantly related to WNV infected dead birds was used to describe the environmental components of the study area. Self-organizing maps found 49 clusters, each of which contained census tracts that were more similar to each other in terms of WNV environmental and socio-economic data. Parallel coordinate plots permitted visualization of each cluster's data, uncovering patterns that allowed final census tract mapping exposing complex spatial patterns contained within the clusters.

Results. Our results suggest that simultaneously visualizing environmental and socio-economic data supports a fuller understanding of the underlying spatial processes for risks to vector-borne disease. Unexpected patterns were revealed in our study that would be useful for developing future multilevel analytical models. For example, when the cluster that contained census tracts with the highest median age was examined, it was determined that those census tracts only contained moderate mosquito habitat risk. Likewise, the cluster that contained census tracts with the highest mosquito habitat risk had populations with moderate median age. Finally, the cluster that contained census tracts with the highest WNV human incidence rates had unexpectedly low mosquito habitat risk.

\section{Introduction}

Variations in infectious disease risk occur across environmental gradients and population groups. Such variations often manifest themselves in geographic space and can be attributed to complex interactions between the environment, population, and behavior (Meade 1977).. The underlying processes behind these interactions occur at different, and often conflicting spatial and temporal scales. Additionally, the data related to those processes are often collected within different spatial boundaries (e.g. county level versus census tract verses ecosystem) and for differing research purposes. Attempts to understand these processes by exploring primary or secondary data sources can introduce additional levels of complexity due to issues of uncertain data collection contexts (Kwan 2012),, incomplete or unavailable data (Zhang and Goodchild 2002), or differences in the underlying questions that drove data collection in the first place (Elliott and Wartenberg 2004).. Further, traditional approaches to model any single complex process of disease risk, including confirmatory or validation steps, rely on well-defined outcome measures and a set of clearly specified dependent and independent variables. In order to explore questions that require coupling more than one complex process simultaneously, traditional modeling techniques do not appear to be directly applicable and will likely need modifications to be useful.

Diez Roux and Mair (2010) show that risk can be expressed at different spatial scales and argue that differential disease risks occur across individual- and group-level characteristics. Individual characteristics include attributes of the individuals at risk (e.g. age, gender, income and other personal attributes) while group-level characteristics include the environmental and socio-economic/demographic context of places to which those populations belong (e.g. vector habitat conditions, socio-economic and demographic profiles, and climatic conditions). Collecting data on these characteristics are driven by various primary questions, measured at various scales, and reported for various purposes. While the disease ecology triangle (Meade 
1977) provides a robust framework for studying interactions between human populations, disease agents, and the environment, it is important to recognize that analytical studies of public health risk must find mechanisms to reconcile process, scale, and data complexity. Some researchers approach this problem with complex multilevel models (multiple scales, misalignment of spatial and temporal boundaries, uncertain research context of the data - see Diez-Roux (2000), (Edsall, MacEachren et al. 2001, Edsall 2003, Edsall 2003, Kraak and Madzudzo 2007). However, in this paper, we suggest that prior to developing these analytical models to combine multiple complex processes, exploratory approaches that use geovisualization techniques may provide valuable insights for identifying variables and associated processes that contribute to variations in disease risk across space and time. These types of insights may prove to be a valuable intermediate step between models that explore environmental determinants of infectious disease risk, or models that explore demographic determinants of infectious disease vulnerability, and more complex models that combine both environmental and demographic variables. We build upon our previous study that used a spatially-explicit environmental model to assess West Nile Virus (WNV) risk in California based on the relationship between WNV incidence and mosquito habitat suitability, and here we report on the visualization of "population" or "human" components of the disease ecology framework (Meade 1977) with a spatial lens. While the environmental data used in our earlier model were of relatively fine geographic resolution (all layers resampled to $120 \mathrm{~m}$ cell size), the study was limited to a county-level analysis due to the non-availability of both fine-scale WNV disease human incidence data and a related, surrogate parameter, WNV infected dead bird data reported at the county level. As many public health researchers lament, our previous analysis would have be more valuable if WNV human incidence data were available at a finer spatial resolution such as census block groups or tract (DeGroote, Sugumaran et al. 2008). None-the-less, we suggest that geovisualization techniques can be used to overcome some of these data limitations by enabling hypothesis generation, seeding confirmatory modeling approaches, and aiding public health practice by providing a platform for exploring complex interactions between the disease, the environment within which it operates, and the populations impacted.

WNV, a vector-borne disease that is primarily spread by the Culex species of mosquitos, was first detected in the United States in 1999 (Nash, Mostashari et al. 2001). Several studies have utilized the information from satellite imagery for environmental characteristics such as temperature, vegetation cover, and moisture (Ozdenerol, Bialkowska-Jelinska et al. 2008) (Rodgers and Mather 2014). Land surface temperature was attributed as one of the main factors contributing to the WNV propagation in Southern California (Liu and Weng 2012). They associated higher temperature to viral replication in mosquitoes and related lower elevations as more susceptible to WNV invasion due to warmer temperatures in plains habitats (Wimberly, Hildreth et al. 2008). Mean temperature during summers, land surface temperature, elevation, diversity of landscape, and water content in vegetation were the main environmental factors contributing to WNV propagation in Southern California. High temperature has been consistently associated with outbreaks and hotspots of WNV activity ((Hartley, Barker et al. 
119

120

121

122

123

124

125

126

127

128

129

130

131

132

133

134

135

136

137

138

139

140

141

142

143

144

145

146

147

148

149

150

151

152

153

154

155

156

157

158

2012, Reisen, Fang et al. 2014, Hoover and Barker 2016),some studies have suggested that certain mosquito species are associated with more urban habitats (Reisen, Takahashi et al. 2008, Kilpatrick 2011, Savage, Anderson et al. 2014), some have linked drought to WNV outbreaks (Paz 2015, Paull, Horton et al. 2017), and others (e.g. Cooke, Grala et al. (2006)) have explored the connections between WNV human infection risk and environmental conditions such as the presence of streams, vegetation, and roads.. Some studies have also suggested mosquito species to be associated with urban habitats, whereas others have linked drought to WNV outbreaks.

In our earlier study (Kala, Tiwari et al. 2017) geostatistical and spatial analysis techniques were used to build a spatially explicit model after exploring multiple environmental factors (i.e. factors directly or indirectly related to known mosquito determinants such as vegetation, elevation, evapotranspiration, streams, land use and temperature) that linked mosquito habitat suitability to the number of WNV-positive dead birds, which was used as a surrogate for human WNV risk. That study concluded that including spatial heterogeneity in the modeling improved predictive ability in understanding WNV risk. A geographically weighted regression (GWR) was applied to a statistically significant ordinary least squares (OLS) model to improve model fit from $61 \%$ to $71 \%$. The resulting WNV disease risk surface was created using multi-criteria decision analysis approach (detailed process can be referred to our previous paper, Kala, Tiwari et al. (2017)). This modeling process was based upon four steps: 1) establishment of the environmental factors, 2) standardization of the factors, 3) establishment of relative weights for each factor, and 4) a Simple Additive Weighting (SAW) method to construct the disease risk surface.

Ruiz, Tedesco et al. (2004) reported that socio-economic factors such as age, income, and race/ethnicity of the human population can also be important predictors of WNV infection risk in humans. While many attempts to predict the risk of WNV transmission have been published, efforts that attempt to link both environmental and socio-economic factors within a spatial framework have resulted in less than complete understanding of the complex relationships associated with human infection risk of WNV. In the study reported here we hypothesize that geovisualization techniques to explore the relationships between disease outcomes, population characteristics, and the environment within which they interact will result in a more complete understanding of the complex patterns related to this disease. A more complete understanding may open doors to developing more traditional model development and model validation approaches that are familiar to public health planners.

This hypothesis suggests that an integrated approach to understanding the relationships of environmental variables and human population demographics on WNV risk should improve our ability to explore large numbers of possible combinations of the processes in order to discover potential hidden but useful patterns. However, Guo, Gahegan et al. (2005) asserted that even in a selected subset of the data it is still a challenge to discover hidden relationships as potential patterns may be expressed in various forms - perhaps linear or non-linear, perhaps spatial or non-spatial, or perhaps some such combination. Geovisualization tools can be useful to support multivariate analysis of geospatial data in order to highlight these potential patterns. We have 
159

160

161

162

163

164

165

166

\section{7}

168

169

170

171

172

173

174

175

176

177

178

179

180

181

182

183

184

185

186

187

188

189

190

191

192

193

194

195

196

197

198

attempted to add value to our earlier GWR model by including information on the spatial characteristics of human population via geovisualization. The addition of demographic data alongside the environmental model may provide understanding to public health planners who want to better understand patterns related to an infectious disease. This added value was accomplished with geovisualization tools to develop self-organizing maps (SOM) and parallel coordinate plots (PCP) to provide insights into the complex processes that operate simultaneously across environmental and socio-economic patterns of this public health issue.

\section{Materials \& Methods}

Disease vectors and pathogen reservoirs typically intersect within the context of specific environmental factors (Rochlin, Turbow et al. 2011), while the risk of host infection is influenced by the composition of a susceptible population. For the mosquito vector, the WNV pathogen and the human host population, environmental and socio-economic factors that have been identified by previous research studies were utilized in this study. Several studies have utilized mosquito habitat suitability as a surrogate for estimating WNV risk for human infection (e.g. Cooke, Grala et al. 2006). In the study reported here, our earlier mosquito habitat suitability model was used to describe the environmental processes occurring in our study area, while census track level demographic data were used to describe the socio-economic processes at play (see Table 1). Figure 1 illustrates the model framework including the advantages of using this approach.

(Table 1 approximately here - environmental and socio-economic factors.)

(Figure 1 approximately here - risk modeling flow chart.)

In the United States, California ranks third in total area (U.S. Census Bureau 2012), and has had the largest population of any state since the 1960's (U.S. Census Bureau 1996, U.S. Census Bureau 2011). There are 58 counties in California, and 8,057 census tracts (U.S. Census Bureau 2019). WNV was first detected in California in 2003 (Reisen, Lothrop et al. 2004), and then received national attention for the high rates of the disease during the following two years (Jean, Honarmand et al. 2007). Results of WNV vector-borne environmental modeling in California (Kala, Tiwari et al. 2017) let to this study of combining socio-economic data with the results of the environmental model using multivariate geovisualization. This study utilized coarse-scale data (county level) of reported cases of WNV human incidence along with infected dead bird counts as the basis for estimating WNV risk. Fine scale environmental (120m pixels) and coarse scale demographic data (census tract level) were used to define environmental and socioeconomic factors for the study area. The study was conducted in two phases: (1) mosquito habitat modeling based on environmental factors and (2) geovisualization techniques based on socio-economic factors. Basemaps for this study were created either using (1) ArcGIS ${ }^{\circledR}$ software by Esri $\left[\operatorname{ArcGIS} \otimes\right.$ and $\operatorname{ArcMap}^{\mathrm{TM}}$ are the intellectual property of Esri and are used herein under 
199 license; copyright (C) Esri; all rights reserved; for more information about Esri® software, please 200 visit http://www.esri.com], or (2) Topologically Integrated Geographic Encoding and

201

202

203

204

205

206

207

208

209

210

211

212

213

214

215

216

217

218

219

220

221

222

223

224

225

226

227

228

229

230

231

232

233

234

235

236

237

238

Referencing system (TIGER) by the U.S. Census Bureau, which is in the Public Domain.

\section{Study Area and environmental and socio-economic factors affecting WNV}

Reported human incidence rates for the study period by county were used to create a 3dimensional database where the $\mathrm{X}$ and $\mathrm{Y}$ dimensions were the geographic centroids of each county, and WNV incidence rates for the county provided the $\mathrm{Z}$ dimension. Those data were then analyzed to generate a spatial 1-standard deviation ellipse (SDE), representing the contiguous region that contained 1-standard deviation of the reported human WNV incidence rates in California. SDE mapping is a common method used to identify spatial direction trends of attribute data associated with geographical features. It has been widely used for geographically identifying disease and crime trends (Chainey, Tompson et al. 2008, Wang, Shi et al. 2015, Leigh, Dunnett et al. 2016, Al-Kindi, Kwan et al. 2017, Ma, Xiao et al. 2017, Polupan, Bezymennyi et al. 2017, Butkovic, Mrdovic et al. 2019, Lu, Deng et al. 2019, Chen, Wang et al. 2020). We used SDE to identify the contiguous region that contained 1-standard deviation of WNV human incidence rates to focus on the counties in California that would most likely reveal previously unknown patterns of WNV risk and vulnerability, and defined that region as our study area.

Once the study area had been determined, socio-economic and environmental data were extracted from each census tract that intersected the ellipse. The dataset contained seven variables for each census tract. A single environmental variable (referred to in this study as "mosquito risk") that represented the results of our earlier GWR model (Kala, Tiwari et al. 2017) was derived from analysis of environmental 8 parameters (stream density, surface temperature, surface slope, cultivated land, developed land, road density, vegetation type, evapotranspiration rate).) Mosquito risk was found to be statistically significantly related to annual WNV-infected dead birds sentinel data, averaged for the 2004-2010 Kala, Tiwari et al. (2017).Annual WNVinfected dead birds sentinel data has been shown to be useful for estimating human WNV risk by multiple studies (Eidson, Komar et al. 2001, Eidson, Kramer et al. 2001, Eidson, Miller et al. 2001, Guptill, Julian et al. 2003, Mostashari, Kulldorff et al. 2003, Ruiz, Tedesco et al. 2004, Johnson, Eidson et al. 2006, Nielsen and Reisen 2007, Patnaik, Juliusson et al. 2007, Chaintoutis, Dovas et al. 2014). The mosquito risk model resulted in a risk surface with a range of 0 to 10 . Higher values indicate higher probability of WNV infected birds based on environmental conditions related to mosquito habitat. For the current study, mosquito risk was extracted for each of the census tract within the study area.

Numerous studies have shown that a susceptible population's risk can be influenced by demographic and socio-economic conditions. For example, Ruiz, Tedesco et al. (2004) and Jean, Honarmand et al. (2007) suggest that the elderly are more susceptible because they have higher rates of weakened immune systems. Males and females may have differing vulnerabilities due to social history or lifestyle (Murray, Baraniuk et al. 2006). Ruiz, Tedesco et al. (2004) also suggest 
239 that race/ethnicity or income influence vulnerability due to behaviors linked to lifestyle. For

240 each census track in the study area, the following data were extracted from 2010 Census data:

241 percent of census tract's population identified as male; percent of census tract's population

242 identified as white; percent of census tract's population identified as black; percent of census

243 tract's population identified as Hispanic; median age of population in census track, and; median

244 household income in census tract.

245

246

247

248

249

250

251

252

253

254

255

256

257

258

259

260

261

262

263

264

265

266

267

268

269

270

271

272

273

274

275

276

277

278

\section{Geovisualization techniques}

This study utilized a spatially explicit exploratory approach for identifying the interaction between different environmental (mosquito habitat) and socio-economic (human demographic) processes occurring in each census tract within a 1-standard deviation ellipse. The approach consisted of utilizing the risk map with multivariate visualization techniques to facilitate the exploration and understanding of complex environmental and socio-economic patterns within the California data. The analysis was facilitated with SomVis, originally an open source Java application, that has now been ported to a web-based service (Brookes, Hernandez-Jover et al. 2014, Fanelli Kuczmarski, Bodt et al. 2018, Mutheneni, Mopuri et al. 2018) (zillioninfo.com). SomVis was/is an integrated software tool consisting of three interactively linked visualizations that can help focus attention on patterns of similarity in complex data sets. The three visualizations used were: (1) a self-organizing map (Kohonen 2001) to perform multivariate analysis, dimensional reduction, and data reduction; (2) a parallel coordinate plot (Inselberg 2002) to visualize the multivariate patterns with display; and (3) geographic mapping (GeoMap) to highlight clusters of specific interrelationships. The geovisualization tools of SOM and PCP have been adopted in many fields of science for exploring difficult high dimensional and nonlinear problems as well as for visualization of multivariate problems (Edsall 2003, Koua and Kraak 2004, Guo, Gahegan et al. 2005, Basara and Yuan 2008, Kaur, Singh et al. 2013, Brookes, Hernandez-Jover et al. 2014, Fanelli Kuczmarski, Bodt et al. 2018, Mutheneni, Mopuri et al. 2018). These tools help to display the high-dimensional datasets, search for hidden relations among the complex set of variables and transform them into a 2-D pattern recognition problem.

Our study highlights the potential of combining these tools along with GIS to detect and analyze different hidden patterns within the complex multivariate data. The coupling of these techniques provides an interesting platform for analyzing larger datasets by integrating it into a spatially-explicit disease model or by using it for near-real time disease monitoring. This user interactive data exploration platform helps identify clusters of complex high dimensional datasets while preserving the topological relationships between data vectors.

I. A self-organizing map (SOM) is used to reduce the dimensionality of data for data visualization purposes while retaining the most information contained within the database. It is a unique partitioning clustering method, which segments multivariate data into nonoverlapping clusters and projects them on a two-dimensional layout. Koua and Kraak (2004) describe SOM as an unsupervised neural clustering technique that is useful in situations 
279

280

281

282

283

284

285

286

287

288

289

290

291

292

293

294

295

296

297

298

299

300

301

302

303

304

305

306

307

308

309

310

311

\section{Results}

313 Our earlier study (Kala, Tiwari et al. 2017) found that the best-fitting mosquito habitat model

\section{4}

315

316

317

318

where the data volumes are large and interrelationships unclear. The approach involves partitioning the dataset where each element (in this case each census tract within the ellipse) is classified into one cluster out of a set number of desired clusters -49 in this study. Clusters contain elements that are similar to each other in terms of the observations for the statistically most relevant variables in the dataset. Some clusters may contain many elements (census tracts), while other may only contain a few, but census tracts within a cluster are more similar to each other than they are to census tracts in other clusters. Likewise, some clusters of census tracts can be more similar to other clusters, but are still different enough to be classified as different clusters according to the feature selection algorithm of SomVis. The clusters are then mapped onto a fixed grid of hexagons, in our case a 13-by-13 grid of hexagons to assist in data visualization. Each cluster is represented with a node (circle) whose diameter is linearly scaled according to the number of census tracts that it contains. Nodes are equally spaced in a two-dimensional space, and behind the nodes is a layer of hexagons, which are shaded to show the multivariate dissimilarity between neighboring nodes. Clusters falling on bright-tone hexagons are more similar to each other than those in darker tones of these hexagons.

II. A parallel coordinate plot (PCP) maps n dimensional space onto a two-dimensional layout by using $\mathrm{n}$ equidistant parallel vertical axes, where $\mathrm{n}$ is the number of variables in the data set. Each vertical axis represents one variable and is linearly scaled using its minimum and maximum values. Each cluster is displayed as a horizontal polyline intersecting each of the vertical axes at the point that corresponds to the respective attribute value for this data element. The thickness of the polyline is proportional to the number of elements in the node (number of census tracts). The PCP can help visualize the data either using combinations of variables (cluster level) or for each individual variable (data item level).

III. Geographic mapping of which census tracts fall within any specific cluster or clusters provides a visual perspective of where the socio-economic and environmental variables of most interest are located. SomVis refers to these as a Geomap and they represent the spatial distribution of multivariate patterns. The Geomap provides a spatial perspective to clusters of similar variables identified using PCPs. These three visual components allow an array of user-controlled interactions that link spatial patterns to the underlying data. that predicted number of WNV infected dead birds in all counties in California had an adjusted $\mathrm{r}^{2}$ of $0.71\left(\mathrm{r}^{2}=0.75, \mathrm{p}<0.05\right)$. Those results agreed with other research (e.g. Beck, Rodriguez et al. 1994) that found that understanding insect borne infectious disease risk is improved when considering spatial heterogeneity of the variables that affect the risk. Our current study, using the same mosquito habitat suitability modeling approach, also found that environmental modeling of

Peer) reviewing PDF | (2020:02:46052:2:0:NEW 24 Jun 2020) 
319

320

321

322

323

324

325

326

327

328

329

330

331

332

333

334

335

336

337

338

339

340

341

342

343

344

345

346

347

348

349

350

351

352

353

354

355

356

357

358

environmental variables is improved when considering spatial heterogeneity of those variables. Figure 2provides a WNV infection risk surface map based on the infected dead bird versus mosquito habitat model.

(Figure 2 approximately here - WNV risk map.)

In this study, we defined our study area as the 1-standard deviation ellipse of reported WNV incidence rates in California. California has 58 counties; 35 counties intersected the ellipse, representing a geographically contiguous area that represents approximately $67 \%$ of all WNV incidence rates. The counties within the ellipse averaged approximately 523,000 hectares in size. Defining this ellipse as our study area was a data reduction approach that allowed focusing on the most relevant WNV incidence rates. Figure 3 represents the counties, color coded by reported incidence rates along, with the 1-standard deviation ellipse based on incidence.

(Figure 3 approximately here - WNV incidence map within the 1-standard deviation ellipse.)

Socio-economic (demographic) variables were extracted for all census tracts within the ellipse. California has 8,040 census tracts, with 1,133 intersecting the 1-standard deviation ellipse. The census tracts within the ellipse averaged 8,780 hectares in size. Environmental and socio-economic data were considered simultaneously with self-organizing map (SOM) analyses. The resultant SOM identified 49 distinct nodes of census tracts (Figure 4).

(Figure 4 approximately here - census tracts within the 1-standard deviation ellipse.)

Each SOM node shown in Figure 5 (indicated with colored circles) represents a cluster of census tracts that are most similar in terms of all 7 variables. The diameter of each node represents the number of census tracts in the node. To illustrate how geovisualization can be used by public health planners, two specific nodes are highlighted for discussion. First, the cluster that contains census tracts with the highest median age is highlighted (labeled as cluster 1 and green in color), and is of interest because it is a variable that has been described as representative of the most vulnerable population (the elderly) to WNV health issues (e.g. Campbell, Marfin et al. (2002)). Second, the cluster that contains census tracts with the highest environmental WNV risk (mosquito habitat) based on the geographically weighted regression model is highlighted (labeled as cluster 2 and blue in color) because of the statistically significant relationship to WNV infected dead bird count.

(Figure 5 approximately here - SOM with 2 clusters highlighted.

Once SOM nodes are defined, a parallel coordinate plot (PCP) can be developed to explore the interaction between different environmental and socio-economic risk factors. The PCP shows 
359

360

361

362

363

364

365

366

367

368

369

370

371

372

373

374

375

376

377

378

379

380

381

382

383

384

385

386

387

388

389

390

391

392

393

394

395

396

397

398

7 vertical axes representing each of the variables under consideration, and 49 polylines representing clusters of census tracts that are most similar to each other for those 7 parameters. Figure 6 represents the PCP with the polyline for cluster 1 (census tracts with the highest average median age) highlighted in green. The PCP indicates that the census tracts contained within this cluster average: (1) the lowest percent male ( $45 \%)$; (2) a moderate household income $(\sim \$ 60,000)$; (3) the lowest percent Hispanic $(\sim 9 \%)$; (4) the highest median age ( $\sim 51$ years); (5) nearly the highest percent white $(\sim 89 \%)$; (6) a low percent black ( 1\%), and; (7) a moderately high mosquito habitat risk $(\sim 6.5)$.

(Figure 6 approximately here - PCP with highest median age.)

Turning to the cluster with census tracts that average the highest environmental risk (mosquito habitat suitability),) this cluster can be visualized with the polyline shown in blue in Figure 7. The PCP indicates that the census tracts contained within this cluster average: (1) a moderate percent male ( $49 \%)$; (2) a moderately low household income $(\sim 55,000)$; (3) a moderately low percent Hispanic ( $19 \%)$; (4) a moderate median age ( $\sim 36$ years); (5) a moderately high percent white ( $79 \%)$; (6) a moderate percent black ( 4\%), and; (7) the highest mosquito habitat risk $(\sim 7.1)$.

(Figure 7 approximately here - PCP with highest mosquito habitat risk highlighted.)

\section{Discussion}

After finding a significant relationship between environmental variables related to Culex mosquito habitat and the number of dead birds infected with WNV, we examined human incidence rates in California to extract socio-economic data (population demographics related to WNV susceptibility).susceptibility Our goal was to use geovisualization techniques to explore the combination of both environmental and socio-economic information to better understand this vector borne infectious disease. Out of the very large number of questions that could be explored with geovisualization, we highlighted two specific ones here: (1) what are the characteristics of the California cluster that represents the census tracts with the highest median age, and; (2) what are the characteristics of the California cluster that represents the census tracts with the highest mosquito habitat risk. Many other questions can be explored once the data are extracted, but to illustrate the technique, we will focus on these two questions.

For example, the cluster that contains the census tracts with the highest median age ( $\sim 58$ years) can be visualized in the SOM - it is the node highlighted in green and labeled "cluster-1" in Figure 5. In the SOM, this node is represented with a circle of moderate diameter indicating that it contains a moderate number of census tracts compared to other nodes, and it is located in a moderately toned gray area indicating that it is moderately dissimilar in multivariate space to other nodes in the study area. "Cluster-2", highlighted in blue in Figure 5, represents the node that contains the census tracts having the highest mosquito habitat risk. The node's diameter is 
399

400

401

402

403

404

405

406

407

408

409

410

411

412

413

414

415

416

417

418

419

420

421

422

423

424

425

426

427

428

429

430

431

432

433

434

435

436

437

438

relatively large, indicating that it contains a large number of census tracts compared to other nodes. Like cluster-1, cluster-2 is located in a moderately toned gray area, indicating moderate dissimilarity to other nodes.

The 49 clusters next were analyzed with parallel cluster plots (PCP), allowing visual inspection of the characteristics of the input parameters of each cluster. Cluster-1 (composition includes highest median age) is highlighted as a green polyline in Figure 6. Following the polyline for cluster-1 indicates that in addition to the highest median age, it also contains a group of census tracts with: the lowest percent males; a moderate median household income; the lowest percent Hispanic; nearly the highest percent white; nearly the lowest percent black; and a moderately high mosquito habitat risk. This visualization may suggest to public health planners that overall this cluster may not be as vulnerable to WNV as the initial reaction for concern for census tracts with the highest median age might imply.

Cluster-2 (environmental context shows highest WNV mosquito habitat risk) is highlighted as a blue polyline in Figure 6. While this group of census tracts represent the highest WNV mosquito habitat risk, they contain relatively moderate levels of the 6 population socio-economic parameters. Implications of the information from this cluster may also be important to inform public health planning.

Clusters can also be viewed spatially for additional geographic insight. Figure 8 provides a map of census tracts with the two highlighted clusters isolated. Census tracts colored green $(n=19)$ represent those with the highest median age. The non-contiguous nature of the census tracts associated with this cluster indicates that they are only similar based on their non-spatial attribute characteristics rather than because of geographical location or autocorrelation. In contrast, the cluster that contains census tracts with the highest WNV mosquito habitat risk (colored blue, $n=30$ ) tend to be concentrated in geographic space. This spatial insight would be valuable to public health planners who may be planning interventions.

(Figure 8 approximately here - highlighted census tracts related to clusters $1 \& 2$ ).

The results from this exploratory analysis suggest that further investigation is required to fully understand the relationship between age and WNV risk. As mentioned above, studies have suggested that elderly people are more vulnerable to WNV, but others such as Carson, Borchardt et al. (2012) shows that WNV infection was greatest for the younger population. It would be simple for public health planners to want to visualize the cluster that contains the census tracts with this composition (lowest median age), and use the PCP to visualize the characteristics of that cluster. If, on the other hand, the planner would rather focus on WNV human incidence rates, census tracts that occur in areas with the highest incidence rates might drive the visualization. For example, Glenn County (near the northern edge of the standard deviation ellipse in Figure 3) reported the highest WNV rate during the study period, so the planner might be interested in finding the cluster(s) that contain the census tracts of this county. This county has 6 census tracts, and Figure 9 shows the PCP highlighting the 5 clusters that contain those 
439 census tracts. Two of the 6 census tracts fall within a single cluster (highlighted in pink), but the

440 other 4 census tracts each fall in 4 separate clusters. These polylines, representing all 5 clusters

441 that occur in the county with the highest WNV incidence rates, reveal an unexpected pattern.

442 These 5 clusters, all representing distinct combinations of environmental and socio-economic

443 data, all have a relatively low WNV mosquito habitat risk. This newly revealed pattern reinforces

444 a suggestion that WNV disease, like other vector-borne infectious diseases, may not necessarily

445 be contracted in the location where a person lives, but rather where they may have traveled to

446 locations that represent higher risk areas. The pathogen may be contracted during outdoor

447 activities in a higher risk area, and then later their disease is diagnosed by the victim's local

448 physician and reported using a local address. While that idea is a common-sense caveat in many

449 vector-borne research conclusions (see for example: Atkinson, Sarkar et al. (2012); (Atkinson,

450 Sarkar et al. 2014); M'ikanatha and Iskander (2014); Riddle (2020)), this data mining

451 geovisualization analysis provides some initial evidence to that effect. The low correspondence

452 between WNV habitat risk (Figure 2) and actual incidence of WNV disease in the population

453 (Figure 9) highlights why a geographically based visualization of the relationships between

454 environmental and socio-economic data may be useful.

455

456

457

458

459

(Figure 9 approximately here - PCP with clusters containing highest incidence rate census tracts)

460

461

462

463

464

465

466

467

468

469

470

471

472

473

474

475

476

477

478

479

Additionally, the public health planner may want to explore all clusters represented in Glenn County in order to understand census tracts outside of Glenn County. For example, if the focus is on the only cluster in Glenn County that contains more than 1 census tract, the planner may want to explore other census tracts outside of Glenn County that are contained in that specific Glenn County cluster. That cluster represents 21 census tracts in the study area (see Figure 10), but they don't have any spatial relationship to each other. After visualizing this pattern, public health practitioners may plan on providing heightened information on detecting WNV symptoms to physicians in those census tracts, since the environmental and socio-economic patterns uncovered in those census tracts are highly related to those in Glenn County, where WNV incidence was the highest.

(Figure 10 approximately here - all census tracts in cluster that contains the 5 Glenn County census tracts)

These examples of geovisualization data mining to explore environmental and socio-economic data related to WNV disease in California represent only a few of the many questions that public health planners may pose. The planners most familiar with the spatial, temporal and historical setting of WNV in California will almost certainly generate different questions. Other infectious diseases in other areas will also generate specific questions to be explored by public health practitioners. Geovisualization will likely provide unique insights. 


\section{Conclusions}

481 Developing new analytical models that combine environmental and socio-economic model for 482 infectious disease planning is difficult because the data are often collected at differing scales, 483 using differing boundaries, and under differing research contexts, each of which might help 484 explain pieces of an infectious disease independently, but in aggregate may provide much better insight. This paper suggests that an exploratory geovisualization process can help planners understand the interplay between environmental and socio-economic data prior to embarking on the difficult development of an analytical model that accounts for these disparities.

This study explored the use of geovisualization techniques to uncover patterns in large, complex data sets that would be difficult to otherwise discover. West Nile Virus was used as a

490

491

492

493

494

495

496

497

498

499

500

501

502

503

504

505

506

507

508

509

510

511

512

513

514

515

516

517

518

519 case study to explore this question - California became the center of United States attention in 2004 and 2005 due to high rate of disease incidence. Geovisualization allowed combining the spatially explicit environmental factors (mosquito habitat risk) with socio-economic data (population demographics) in a data mining context to find previously unknown data clusters at the census tract level. Major challenges for multivariate geospatial mapping include large data volumes, high dimensionality, and the perception of complex patterns (Guo 2009). The research reported here utilizes a spatially explicit exploratory approach that combines geovisualization, spatial analysis, and computational methods for identifying the interaction between different environmental and socio-economic factors. There are multi-level dynamics involved in a disease transmission including complex environmental procedures and the population dynamics. Our research has explored the use of spatially explicit geovisualization techniques for identification of interesting clusters (based on their multivariate similarity) for future investigation. Our results suggest that the visualization of similarity clustering of multivariate attributes facilitates the analysis of complex data. It also helps expose the underlying spatial processes that may result in differential risks. Another advantage of this approach is that patterns found in voluminous and complex epidemiological data can provide more focused opportunities for analysis and interpretation by experts in that field. With an interactive user platform, geovisualization techniques can efficiently obtain new knowledge from the data and become an important hypothesis-generating tool in public health research. Understanding underlying environmental and socio-economic characteristics for the occurrence of WNV, or any infectious disease, is important for mitigating future outbreaks.

We have shown a few examples of how geovisualization could be used by public health planners to better understand and respond to an infectious disease outbreak. This approach found 1,133 census tracts within our study area of WNV incidence in California, and classified those census tracts into 49 clusters where each cluster contained census tracts that were more similar to each other in terms of WNV environmental and socio-economic parameters, than to the census tracts represented in all other clusters. Examples of several interesting patterns were revealed. For example, the cluster that had census tracts with the highest average mosquito habitat risk only had mid-level median age levels. Had there been a cluster that had both the highest mosquito habitat risk and the highest median age, public health planners might choose more 
520 intense intervention measures in those census tracts. Another interesting pattern uncovered was

521 that census tracts in the county that had the highest reported incidence of WNV had relatively

522 low mosquito habitat risk. This might lead to a speculation that demographic and sociosocio-

523 economic parameters should be weighted more importantly than mosquito habitat risk when

524 developing public health plans. Likewise, this pattern might suggest other factors like poor links

525 between modelled mosquito habitat risk and WNV risk in areas outside the training set data or

526 spatial biases in recording effort operating differently at the county level and the census tract

527 level could be at play. Focusing on those ideas through geovisualization may reveal other

528 unknown patterns.

529 This paper represents a case study that utilized a retrospective view of a WNV outbreak in

530 California in the mid 2000's. At that time, geovisualization tools were quite limited and not often

531 used by public health practitioners. Now that the tools are more available, and much easier to

532 use, a future research program that explores using geovisualization in near-real time during an

533 outbreak is appropriate. Infectious disease outbreaks occur frequently, and rapid planning and

534 response are always desirable. Many of these outbreaks are not well understood, and adequate

535 interventions could certainly benefit from data mining, geovisualization approaches. For

536 example, at the time of this writing the Coronavirus (COVID-19) was first reported to the public

537 on 31 December 2019, after the outbreak was first detected in Wuhan City, China (CDC 2020).

538 By mid-February 2020, tens of thousands of cases were reported and news of the virus spreading 539 outside of China started appearing in January 2020. This outbreak will clearly create a large and

540 complex dataset, and public health planners would certainly benefit if they were able to explore

541 geospatial patterns in that dataset in near-real time.

542

543 Acknowledgements

544 The authors would like to acknowledge the pain and suffering of victims of West Nile Virus, as

545 well as all other vector-borne infectious diseases.

546

547 References

548

549

550

551

552

553

554

555

556

557

558

559

560

Al-Kindi, K. M., P. Kwan, N. R. Andrew and M. Welch (2017). "Modelling spatiotemporal patterns of dubas bug infestations on date palms in northern Oman: A geographical information system case study." Crop protection 93: 113-121.

Atkinson, S. F., S. Sarkar, A. Aviña, J. A. Schuermann and P. Williamson (2012). "Modelling spatial concordance between Rocky Mountain spotted fever disease incidence and habitat probability of its vector Dermacentor variabilis (American dog tick)." Geospatial Health 7(1): 91-100.

Atkinson, S. F., S. Sarkar, A. Aviña, J. A. Schuermann and P. Williamson (2014). "A determination of the spatial concordance between Lyme disease incidence and habitat probability of its primary vector Ixodes scapularis (black-legged tick)." Geospatial health: 203-212.

Basara, H. G. and M. Yuan (2008). "Community health assessment using self-organizing maps and geographic information systems." International journal of health geographics 7(1): 67. 
561

562

563

564

565

566

567

568

569

570

571

572

573

574

575

576

577

578

579

580

581

582

583

584

585

586

587

588

589

590

591

592

593

594

595

596

597

598

599

600

601

602

603

604

605

606

Beck, L. R., M. H. Rodriguez, S. W. Dister, A. D. Rodriguez, E. Rejmankova, A. Ulloa, R. A. Meza, D. R. Roberts, J. F. Paris and M. A. Spanner (1994). "Remote sensing as a landscape epidemiologic tool to identify villages at high risk for malaria transmission." The American journal of tropical medicine and hygiene 51(3): 271-280.

Brookes, V., M. Hernandez-Jover, R. Neslo, B. Cowled, P. Holyoake and M. P. Ward (2014). "Identifying and measuring stakeholder preferences for disease prioritisation: a case study of the pig industry in Australia." Preventive veterinary medicine 113(1): 118-131.

Butkovic, A., S. Mrdovic, S. Uludag and A. Tanovic (2019). "Geographic profiling for serial cybercrime investigation." Digital Investigation 28: 176-182.

Campbell, G. L., A. A. Marfin, R. S. Lanciotti and D. J. Gubler (2002). "West nile virus." The Lancet infectious diseases 2(9): 519-529.

Carson, P. J., S. M. Borchardt, B. Custer, H. E. Prince, J. Dunn-Williams, V. Winkelman, L. Tobler, B. J. Biggerstaff, R. Lanciotti, L. R. Petersen and M. P. Busch (2012). "Neuroinvasive disease and West Nile virus infection, North Dakota, USA, 1999-2008." Emerging infectious diseases 18(4): 684-686.

CDC. (2020). "Coronavirus Disease 2019(COVID-19) Situation Summary " Retrieved 18 February, 2020, from https://www.cdc.gov/coronavirus/2019-ncov/summary.html.

Chainey, S., L. Tompson and S. Uhlig (2008). "The utility of hotspot mapping for predicting spatial patterns of crime." Security journal 21(1-2): 4-28.

Chaintoutis, S. C., C. I. Dovas, M. Papanastassopoulou, S. Gewehr, K. Danis, C. Beck, S. Lecollinet, V. Antalis, S. Kalaitzopoulou and T. Panagiotopoulos (2014). "Evaluation of a West Nile virus surveillance and early warning system in Greece, based on domestic pigeons." Comparative immunology, microbiology and infectious diseases 37(2): 131-141.

Chen, J., J. Wang, M. Wang, R. Liang, Y. Lu, Q. Zhang, Q. Chen and B. Niu (2020). "Retrospect and Risk Analysis of Foot-and-Mouth Disease in China Based on Integrated Surveillance and Spatial Analysis Tools." Frontiers in Veterinary Science 6: 511.

Cooke, W. H., K. Grala and R. C. Wallis (2006). "Avian GIS models signal human risk for West Nile virus in Mississippi." International Journal of Health Geographics 5(1): 36.

DeGroote, J. P., R. Sugumaran, S. M. Brend, B. J. Tucker and L. C. Bartholomay (2008). "Landscape, demographic, entomological, and climatic associations with human disease incidence of West Nile virus in the state of Iowa, USA." International Journal of Health Geographics 7(1): 19.

Diez Roux, A. V. and C. Mair (2010). "Neighborhoods and health." Annals of the New York Academy of Sciences 1186(1): 125-145.

Diez-Roux, A. V. (2000). "Multilevel analysis in public health research." Annual review of public health 21(1): 171-192.

Edsall, R. M. (2003). "Design and usability of an enhanced geographic information system for exploration of multivariate health statistics." The Professional Geographer 55(2): 146-160.

Edsall, R. M. (2003). "The parallel coordinate plot in action: design and use for geographic visualization." Computational Statistics \& Data Analysis 43(4): 605-619.

Edsall, R. M., A. M. MacEachren and L. Pickle (2001). Case Study: Design and Assessment of an Enhanced Geographic Information System for Exploration of Multivariate Health Statistics. infovis.

Eidson, M., N. Komar, F. Sorhage, R. Nelson, T. Talbot, F. Mostashari, R. McLean and W. N. V. A. M. S. Group (2001). "Crow deaths as a sentinel surveillance system for West Nile virus in the northeastern United States, 1999." Emerging infectious diseases 7(4): 615.

Peer] reviewing PDF | (2020:02:46052:2:0:NEW 24 Jun 2020) 
607 Eidson, M., L. Kramer, W. Stone, Y. Hagiwara, K. Schmit and N. Y. S. W. N. V. A. S. Team

608 (2001). "Dead bird surveillance as an early warning system for West Nile virus." Emerging $609 \quad$ infectious diseases 7(4): 631.

610 Eidson, M., J. Miller, L. Kramer, B. Cherry, Y. Hagiwara and W. N. V. B. M. A. Group (2001).

611

612

613

614

615

616

617

618

619

620

621

622

623

624

625

626

627

628

629

630

631

632

633

634

635

636

637

638

639

640

641

642

643

644

645

646

647 "Dead crow densities and humat
infectious diseases 7(4): 662 .

Elliott, P. and D. Wartenberg (2004). "Spatial epidemiology: current approaches and future challenges Environ Health Perspect 112: 998-1006." Find this article online.

Fanelli Kuczmarski, M., B. A. Bodt, E. Stave Shupe, A. B. Zonderman and M. K. Evans (2018). "Dietary patterns associated with lower 10-year atherosclerotic cardiovascular disease risk among urban African-American and white adults consuming western diets." Nutrients 10(2): 158.

Guo, D. (2009). "Multivariate Spatial Clustering and Geovisualization." Geographic Data Mining and Knowledge Discovery: 325.

Guo, D., M. Gahegan, A. M. MacEachren and B. Zhou (2005). "Multivariate analysis and geovisualization with an integrated geographic knowledge discovery approach." Cartography and Geographic Information Science 32(2): 113-132.

Guptill, S. C., K. G. Julian, G. L. Campbell, S. D. Price and A. A. Marfin (2003). "Early-season avian deaths from West Nile virus as warnings of human infection." Emerging infectious diseases 9(4): 483.

Hartley, D. M., C. M. Barker, A. Le Menach, T. Niu, H. D. Gaff and W. K. Reisen (2012). "Effects of temperature on emergence and seasonality of West Nile virus in California." The American journal of tropical medicine and hygiene 86(5): 884-894.

Hoover, K. C. and C. M. Barker (2016). "West Nile virus, climate change, and circumpolar vulnerability." Wiley Interdisciplinary Reviews: Climate Change 7(2): 283-300.

Inselberg, A. (2002). "Visualization and data mining of high-dimensional data." Chemometrics and intelligent laboratory systems 60(1): 147-159.

Jean, C. M., S. Honarmand, J. K. Louie and C. A. Glaser (2007). "Risk factors for West Nile virus neuroinvasive disease, California, 2005." Emerging infectious diseases 13(12): 1918.

Johnson, G. D., M. Eidson, K. Schmit, A. Ellis and M. Kulldorff (2006). "Geographic prediction of human onset of West Nile virus using dead crow clusters: an evaluation of year 2002 data in New York State." American journal of epidemiology 163(2): 171-180.

Kala, A. K., C. Tiwari, A. R. Mikler and S. F. Atkinson (2017). "A comparison of least squares regression and geographically weighted regression modeling of West Nile virus risk based on environmental parameters." PeerJ 5: e3070.

Kaur, A., N. Singh and A. Bahrdwaj (2013). "A comparison of supervised multilayer back propagation and unsupervised self organizing maps for the diagnosis of thyroid disease." International Journal of Computer Applications 82(13).

Kilpatrick, A. M. (2011). "Globalization, land use, and the invasion of West Nile virus." Science 334(6054): 323-327.

648 Koua, E. L. and M.-J. Kraak (2004). "Geovisualization to support the exploration of large health

649

650

651

652 and demographic survey data." International journal of health geographics 3(1): 12.

Kraak, M.-J. and P. Madzudzo (2007). Space time visualization for epidemiological research. ICC 2007: Proceedings of the 23nd international cartographic conference ICC: Cartography for everyone and for you, International Cartographic Association.

Peer] reviewing PDF | (2020:02:46052:2:0:NEW 24 Jun 2020) 
653 Kwan, M.-P. (2012). "The uncertain geographic context problem." Annals of the Association of

654

655

656

657

658

659

660

661

662

663

664

665

666

667

668

669

670

671

672

673

674

675

676

677

678

679

680

681

682

683

684

685

686

687

688

689

690

691

692

693

694

695

696 American Geographers 102(5): 958-968.

Leigh, J. M., S. J. Dunnett and L. M. Jackson (2016). Predictive policing using hotspot analysis. Proceedings of the international multiconference of engineers and computer scientists.

Liu, H. and Q. Weng (2012). "Enhancing temporal resolution of satellite imagery for public health studies: a case study of West Nile Virus outbreak in Los Angeles in 2007." Remote Sensing of Environment 117: 57-71.

Lu, Y., X. Deng, J. Chen, J. Wang, Q. Chen and B. Niu (2019). "Risk analysis of African swine fever in Poland based on spatio-temporal pattern and Latin hypercube sampling, 2014-2017." BMC veterinary research 15(1): 160 .

M'ikanatha, N. M. and J. K. Iskander (2014). "Surveillance as a foundation for infectious disease prevention and control." Concepts and Methods in Infectious Disease Surveillance: 1-6.

Ma, J., J. Xiao, X. Gao, B. Liu, H. Chen and H. Wang (2017). "Spatial pattern of foot-and-mouth disease in animals in China, 2010-2016." PeerJ 5: e4193.

Meade, M. S. (1977). "Medical geography as human ecology: the dimension of population movement." Geographical Review: 379-393.

Mostashari, F., M. Kulldorff, J. J. Hartman, J. R. Miller and V. Kulasekera (2003). "Dead bird clusters as an early warning system for West Nile virus activity." Emerging infectious diseases 9(6): 641 .

Murray, K., S. Baraniuk, M. Resnick, R. Arafat, C. Kilborn, K. Cain, R. Shallenberger, T. York, D. Martinez and J. Hellums (2006). "Risk factors for encephalitis and death from West Nile virus infection." Epidemiology and infection 134(06): 1325-1332.

Mutheneni, S. R., R. Mopuri, S. Naish, D. Gunti and S. M. Upadhyayula (2018). "Spatial distribution and cluster analysis of dengue using self organizing maps in Andhra Pradesh, India, 2011-2013." Parasite epidemiology and control 3(1): 52-61.

Nash, D., F. Mostashari, A. Fine, J. Miller, D. O'Leary, K. Murray, A. Huang, A. Rosenberg, A. Greenberg and M. Sherman (2001). "The outbreak of West Nile virus infection in the New York City area in 1999." New England Journal of Medicine 344(24): 1807-1814.

Nielsen, C. F. and W. K. Reisen (2007). "West Nile virus-infected dead corvids increase the risk of infection in Culex mosquitoes (Diptera: Culicidae) in domestic landscapes." Journal of medical entomology 44(6): 1067-1073.

Ozdenerol, E., E. Bialkowska-Jelinska and G. N. Taff (2008). "Locating suitable habitats for West Nile Virus-infected mosquitoes through association of environmental characteristics with infected mosquito locations: a case study in Shelby County, Tennessee." International Journal of Health Geographics 7: 12.

Patnaik, J. L., L. Juliusson and R. L. Vogt (2007). "Environmental predictors of human West Nile virus infections, Colorado." Emerging infectious diseases 13(11): 1788.

Paull, S. H., D. E. Horton, M. Ashfaq, D. Rastogi, L. D. Kramer, N. S. Diffenbaugh and A. M. Kilpatrick (2017). "Drought and immunity determine the intensity of West Nile virus epidemics and climate change impacts." Proceedings of the Royal Society B: Biological Sciences 284(1848): 20162078.

Paz, S. (2015). "Climate change impacts on West Nile virus transmission in a global context." Philosophical Transactions of the Royal Society B: Biological Sciences 370(1665): 20130561. 
697 Polupan, I., M. Bezymennyi, M. Golik, Z. Drozhzhe, S. Nychyk and V. Nedosekov (2017).

698

699

700

701

702

703

704

705

706

707

708

709

710

711

712

713

714

715

716

717

718

719

720

721

722

723

724

725

726

727

728

729

730

731

732

733

734

735

736

737

738
"Spatial and temporal patterns of enzootic rabies on the territory of Chernihiv oblast of Ukraine." Journal for veterinary medicine, biotechnology and biosafety(3, Iss. 2): 31-36.

Reisen, W., H. Lothrop, R. Chiles, M. Madon, C. Cossen, L. Woods, S. Husted, V. Kramer and J. Edman (2004). "West Nile virus in California." Emerging infectious diseases 10(8): 1369.

Reisen, W. K., Y. Fang and V. M. Martinez (2014). "Effects of temperature on the transmission of West Nile virus by Culex tarsalis (Diptera: Culicidae)." Journal of medical entomology 43(2): 309-317.

Reisen, W. K., R. M. Takahashi, B. D. Carroll and R. Quiring (2008). "Delinquent mortgages, neglected swimming pools, and West Nile virus, California." Emerging infectious diseases 14(11): 1747.

Riddle, M. S. (2020). "Travel, Diarrhea, Antibiotics, Antimicrobial Resistance and Practice Guidelines-a Holistic Approach to a Health Conundrum." Current Infectious Disease Reports 22(4): 1-10.

Rochlin, I., D. Turbow, F. Gomez, D. V. Ninivaggi and S. R. Campbell (2011). "Predictive mapping of human risk for West Nile virus (WNV) based on environmental and socioeconomic factors." PloS one 6(8): e23280.

Rodgers, S. E. and T. N. Mather (2014). "Evaluating Satellite Sensor-Derived Indices for Lyme Disease Risk Prediction." Journal of Medical Entomology 43(2): 337-343.

Ruiz, M. O., C. Tedesco, T. J. McTighe, C. Austin and U. Kitron (2004). "Environmental and social determinants of human risk during a West Nile virus outbreak in the greater Chicago area, 2002." International Journal of Health Geographics 3(1): 8.

Savage, H. M., M. Anderson, E. Gordon, L. Mcmillen, L. Colton, M. Delorey, G. Sutherland, S. Aspen, D. Charnetzky and K. Burkhalter (2014). "Host-seeking heights, host-seeking activity patterns, and West Nile virus infection rates for members of the Culex pipiens complex at different habitat types within the hybrid zone, Shelby County, TN, 2002 (Diptera:

Culicidae)." Journal of medical entomology 45(2): 276-288.

U.S. Census Bureau (1996). Population of States and Counties of the United States: 1790-1990. Washington D.C., U.S. Department of Commerce: 236.

U.S. Census Bureau (2011). Population Distribution and Change: 2000-2010. Washington D.C., U.S. Department of Commerce: 12.

U.S. Census Bureau (2012). United States Summary: 2010, Population and Housing Unit Counts. Washington D.C., U.S. Department of Commerce: 554.

U.S. Census Bureau (2019). Guide to State and Local Census Geography. Washington D.C., U.S. Department of Commerce: 191.

Wang, B., W. Shi and Z. Miao (2015). "Confidence analysis of standard deviational ellipse and its extension into higher dimensional Euclidean space." PloS one 10(3).

Wimberly, M. C., M. B. Hildreth, S. P. Boyte, E. Lindquist and L. Kightlinger (2008).

"Ecological niche of the 2003 West Nile virus epidemic in the northern Great Plains of the United States." PloS one 3(12): e3744.

Zhang, J. and M. F. Goodchild (2002). Uncertainty in geographical information, CRC press. 


\section{Table 1 (on next page)}

Variables related to susceptible human population characteristics (composition) and vector habitat characteristics (context) utilized in this study. 
2

\begin{tabular}{|l|l|l|l|}
\hline \multicolumn{2}{|c|}{$\begin{array}{c}\text { Human population characteristics } \\
\text { (demographic composition) }\end{array}$} & \multicolumn{1}{c|}{$\begin{array}{c}\text { Mosquito habitat characteristics } \\
\text { (environmental context) }\end{array}$} \\
\hline $\begin{array}{c}\text { Factors studied } \\
\text { (reference) }\end{array}$ & $\begin{array}{c}\text { Relation to WNV } \\
\text { risk }\end{array}$ & \multicolumn{1}{c|}{$\begin{array}{c}\text { Factors studied } \\
\text { (reference) }\end{array}$} & \multicolumn{1}{c|}{ Relation to WNV risk } \\
\hline $\begin{array}{l}\text { Old age } \\
\text { (Jean et al. 2007; } \\
\text { Ruiz et al. 2004) }\end{array}$ & $\begin{array}{l}\text { Weakened immune } \\
\text { system }\end{array}$ & $\begin{array}{l}\text { Stream, Vegetation, } \\
\text { Road } \\
\text { (Cooke et al. 2006; } \\
\text { Kala et al. 2017) }\end{array}$ & Sites for breeding and resting. \\
\hline $\begin{array}{l}\text { Male sex } \\
\text { (Murray et al. 2006) }\end{array}$ & $\begin{array}{l}\text { Social history or } \\
\text { lifestyle. }\end{array}$ & $\begin{array}{l}\text { Temperature } \\
\text { (Kala et al. 2017; } \\
\text { Wimberly et al. 2008) }\end{array}$ & $\begin{array}{l}\text { Increases growth rate of vector, } \\
\text { decreases egg development cycle } \\
\text { and shortens extrinsic incubation } \\
\text { period of vector. }\end{array}$ \\
\hline $\begin{array}{l}\text { Race/Ethnicity } \\
\text { (Ruiz et al. 2004) }\end{array}$ & $\begin{array}{l}\text { Increased risk from } \\
\text { behaviors linked to } \\
\text { their lifestyle. }\end{array}$ & $\begin{array}{l}\text { Surface slope } \\
\text { (Ozdenerol et al. 2008) }\end{array}$ & $\begin{array}{l}\text { Water stagnation creating } \\
\text { mosquito breeding ground. }\end{array}$ \\
\hline $\begin{array}{l}\text { Income } \\
\text { (Ruiz et al. 2004) }\end{array}$ & $\begin{array}{l}\text { Increased risk from } \\
\text { behaviors linked to } \\
\text { their lifestyle. }\end{array}$ & $\begin{array}{l}\text { Cultivated land, } \\
\text { Developed land } \\
\text { (Kilpatrick 2011) }\end{array}$ & $\begin{array}{l}\text { Preferred natural ground pools in } \\
\text { cultivated land and warmer micro- } \\
\text { climates in developed lands. }\end{array}$ \\
\hline
\end{tabular}

3

4 
Figure 1

West Nile Virus (WNV) risk and susceptibility geovisualization modeling framework.

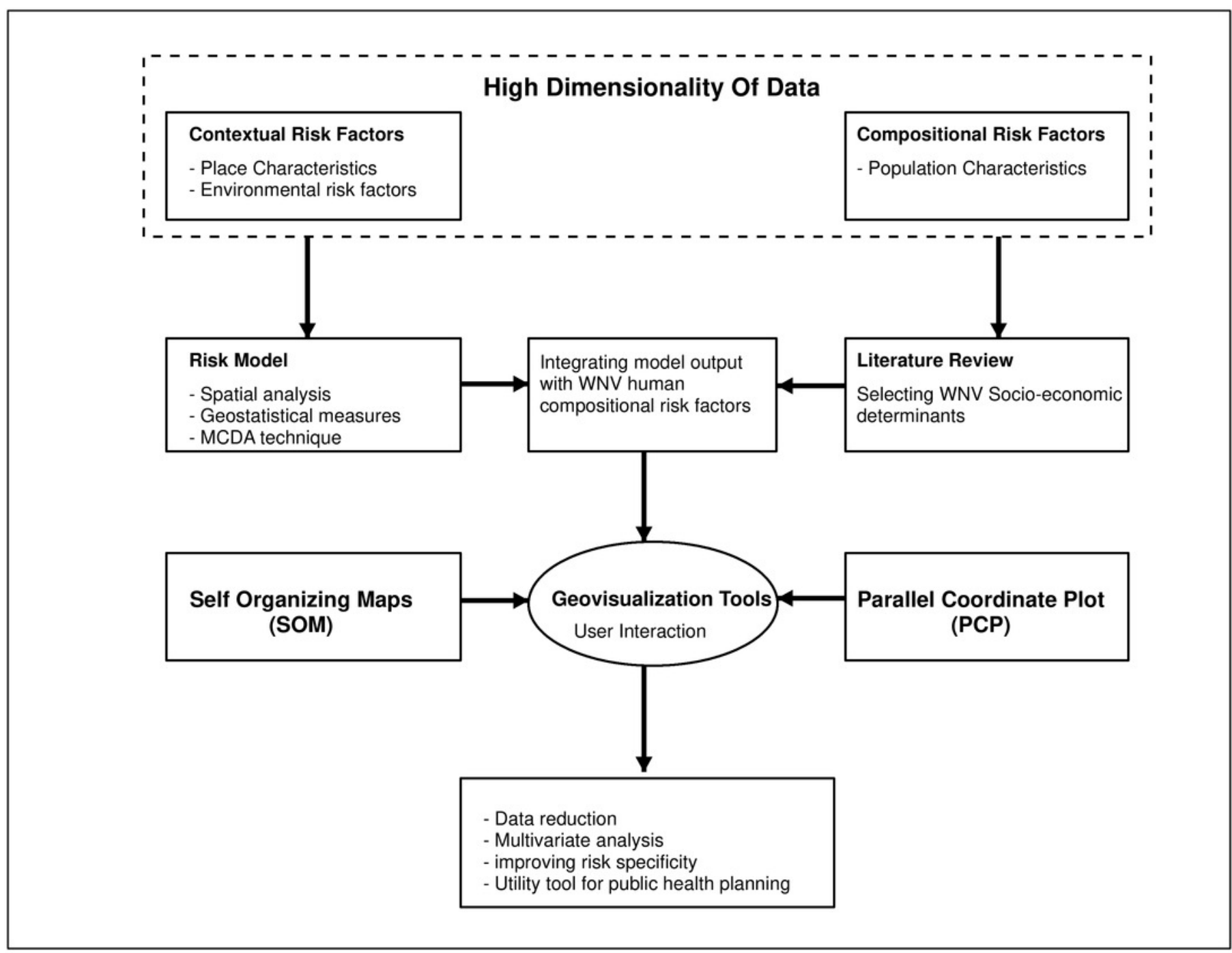


Figure 2

West Nile Virus (WNV) risk based on environmental context modeling (i.e. mosquito habitat risk).

Risk is represented by a unitless value that can theoretically range from a low of 0 (zero) to a high of 10 (ten), based on environmental variables that linked mosquito habitat to WNV infected dead birds as described in Kala, Tiwari, Mikler and Atkinson, 2017). 


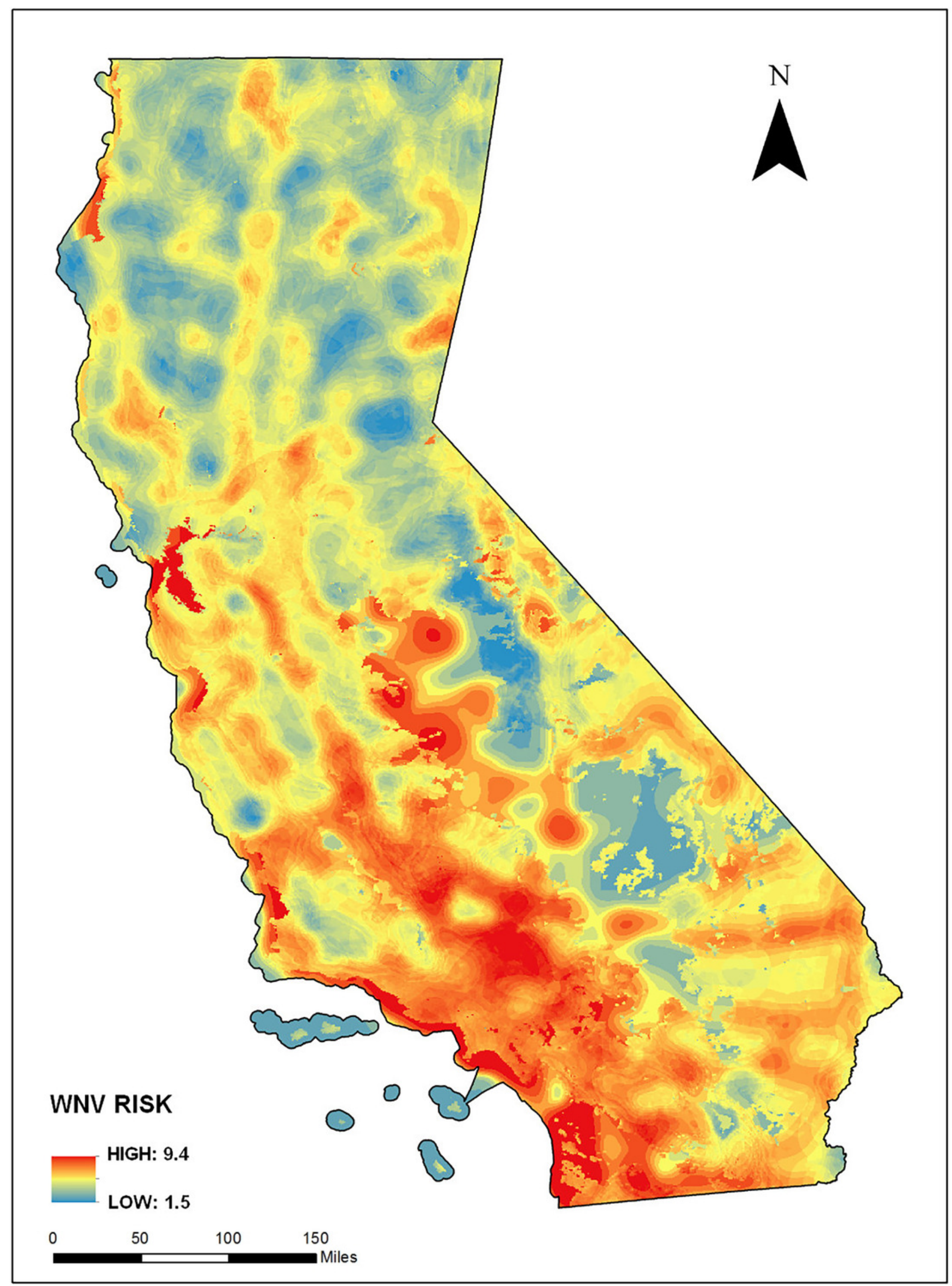




\section{Figure 3}

West Nile Virus (WNV) human incidence rate by county with a 1-standard deviation ellipse superimposed.

California has 58 counties; 31 counties are contained within or intersect with the 1-standard deviation ellipse. Colors represent quintiles of reported human incidence of WNV. Built using ESRI ArcGIS ${ }^{\circledR}$ and ArcMap $^{T M}$ basemap files (ESRI, Redlands, CA, USA). Sources for basemap: National Geographic, Esri, Garmin, HERE, UNEP-WCMC, USGS, NASA, ESA, METI, NRCAN, GEBCO, NOAA, increment P Corp. 


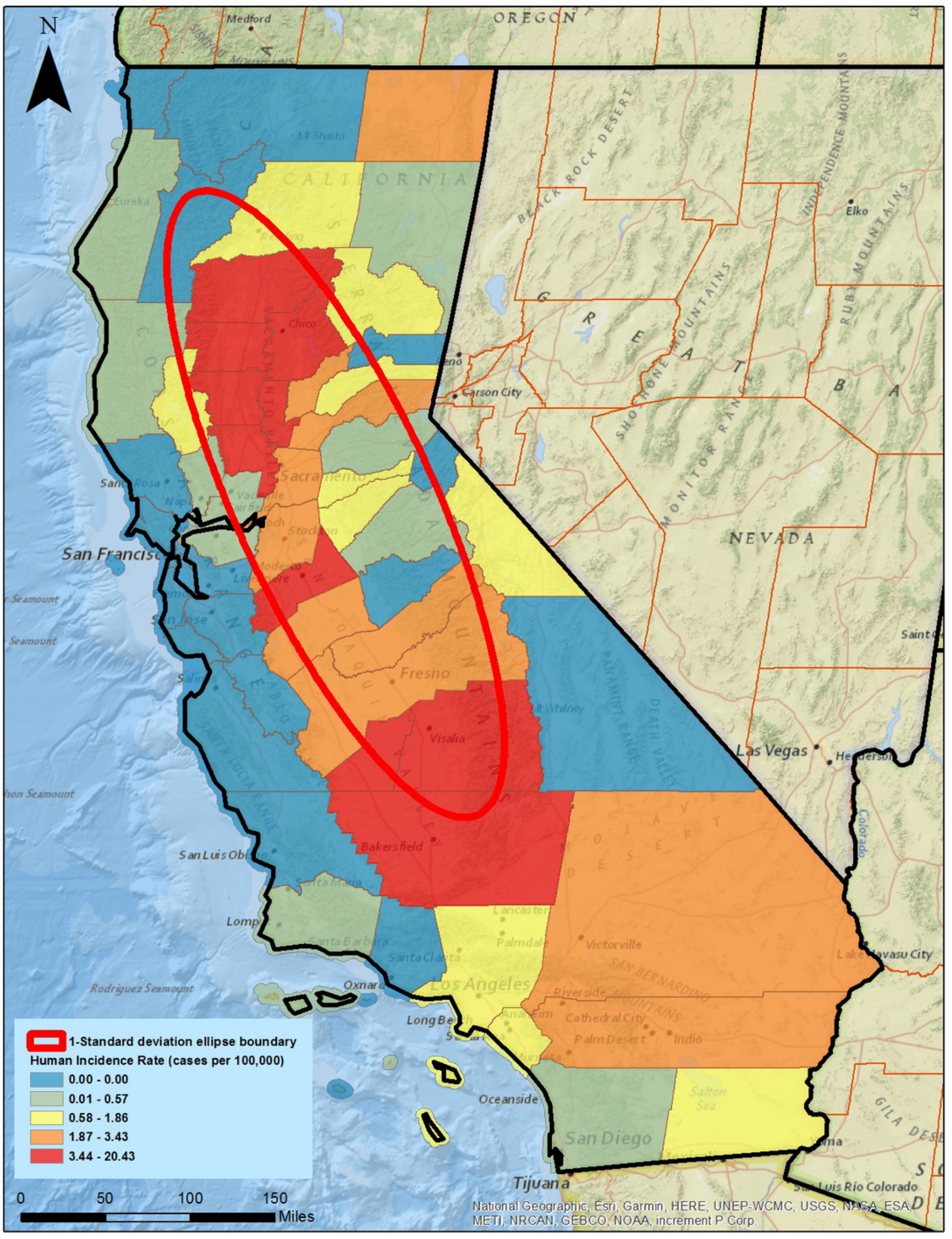


Figure 4

Census tracts $(1,133)$ within the 1-standard deviation ellipse of human West Nile Virus (WNV) incidence rate.

Built using ESRI ArcGIS ${ }^{\circledR}$ and $\operatorname{ArcMap}^{\mathrm{TM}}$ basemap files (ESRI, Redlands, CA, USA) and Topologically Integrated Geographic Encoding and Referencing system (Tiger) by U.S. Census Bureau. Sources for basemap: National Geographic, Esri, Garmin, HERE, UNEP-WCMC, USGS, NASA, ESA, METI, NRCAN, GEBCO, NOAA, increment P Corp, U.S. Census Bureau. 


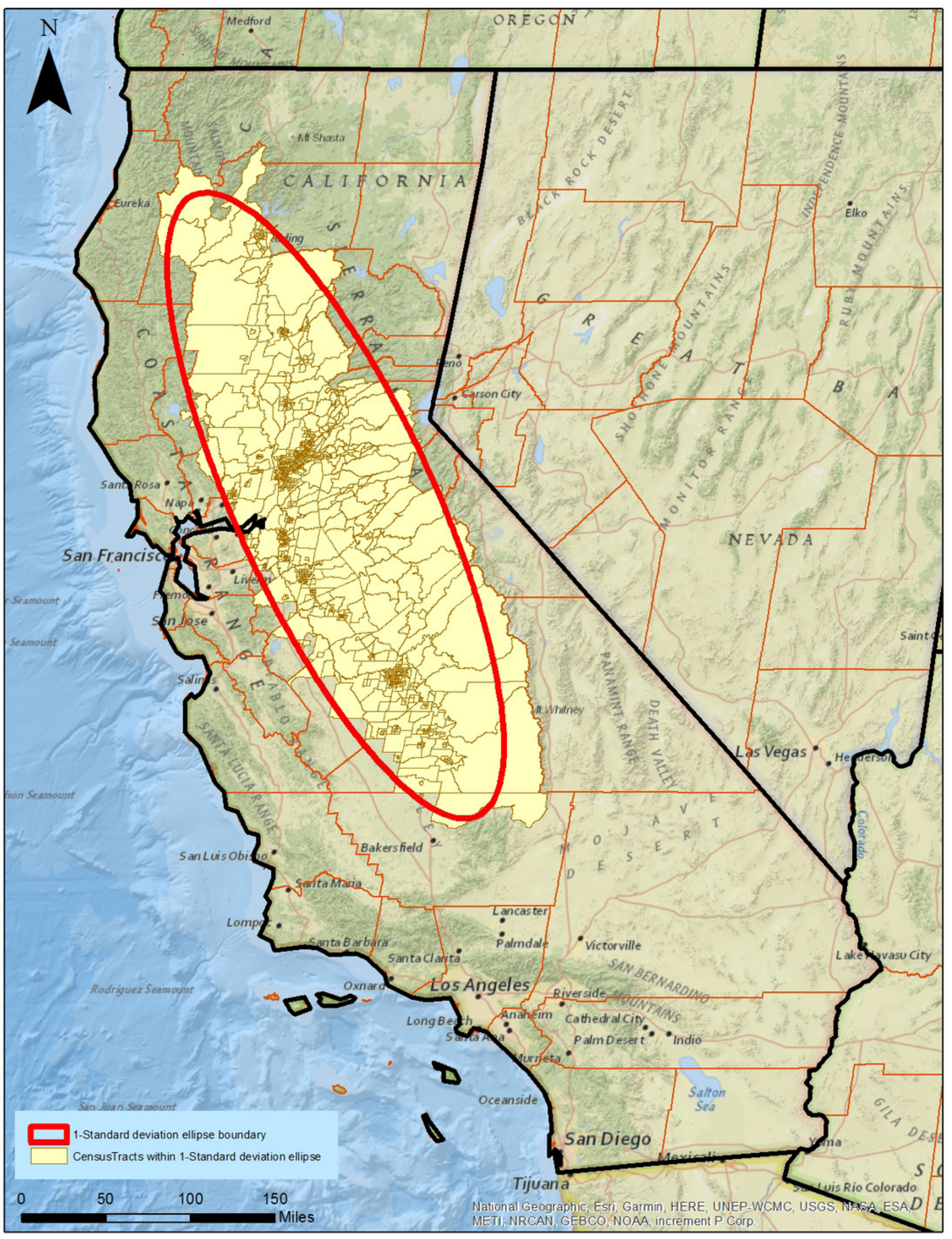




\section{Figure 5}

Self Organizing Map (SOM) representing 49 nodes with valid combination of contextual and compositional parameters from 1,133 census tracts.

Size of node (circle) reflects how many census tracts in the cluster. Darker gray shading of background hexagons represents more dissimilarity to nearby clusters

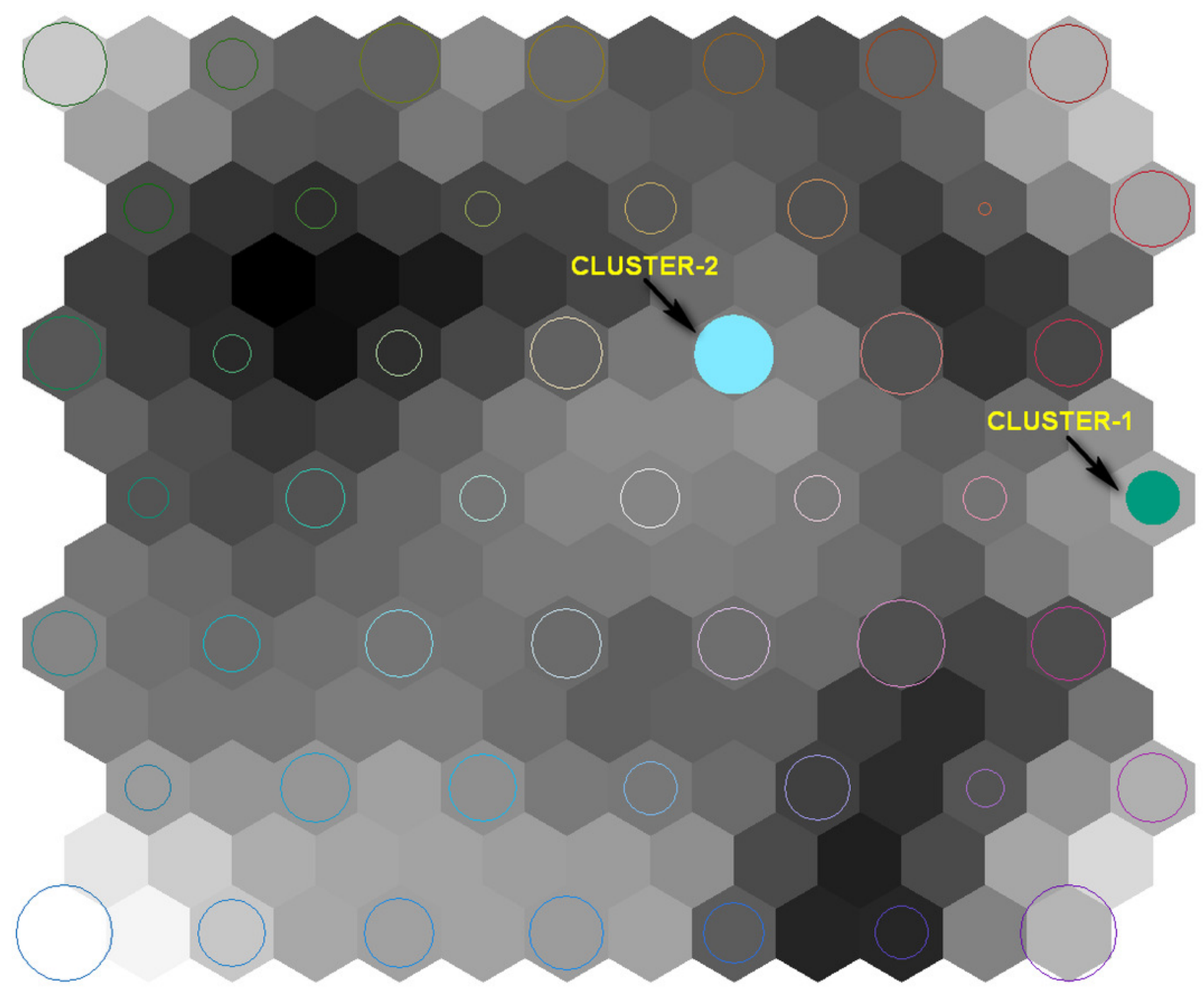




\section{Figure 6}

Parallel Coordinate Plot (PCP) showing 49 polylines representing each cluster; green highlighted polyline represents cluster with the highest median age.

Compositional parameters include average values of all census tracts in cluster for: percent of population that is male, median household income, percent Hispanic, median age, percent white, percent black. Contextual parameters include mosquito habitat risk based on environmental parameters related to West Nile Virus infected dead birds. Bold numbers on each axis represent the maximum average value and the minimum average value for the 49 clusters.

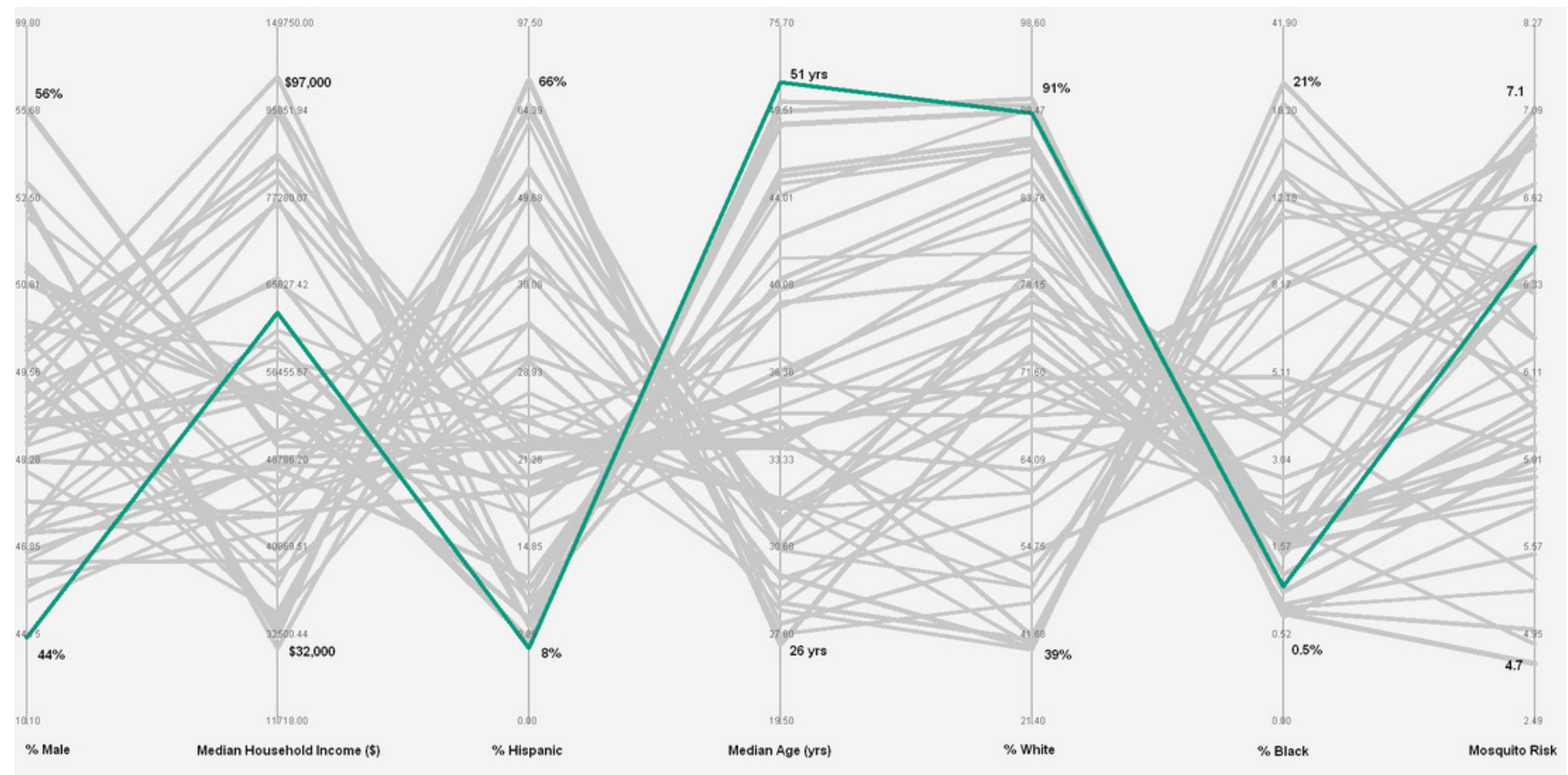


Figure 7

Parallel Coordinate Plot (PCP) showing 49 polylines representing each cluster; blue highlighted polyline represents cluster with the highest mosquito habitat risk.

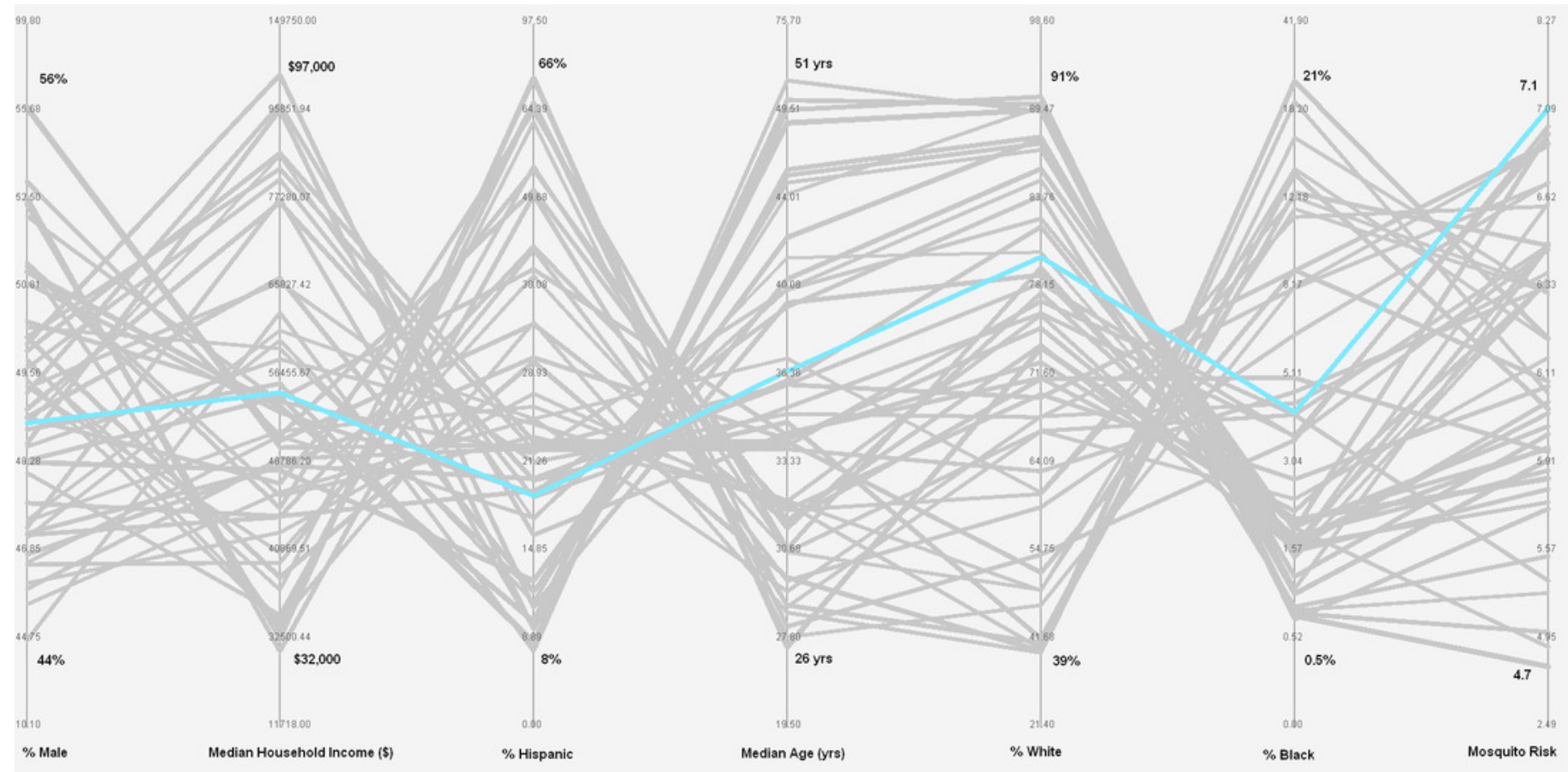




\section{Figure 8}

Geomap showing spatial context of census tracts contained in the cluster (\#1 in the SOM) with the highest median age (green) and the census tracts in the cluster (\#2 in the SOM) with the highest mosquito habitat risk (blue).

Built using Topologically Integrated Geographic Encoding and Referencing system (TIGER) basemap files. Sources for basemap: U.S. Census Bureau.

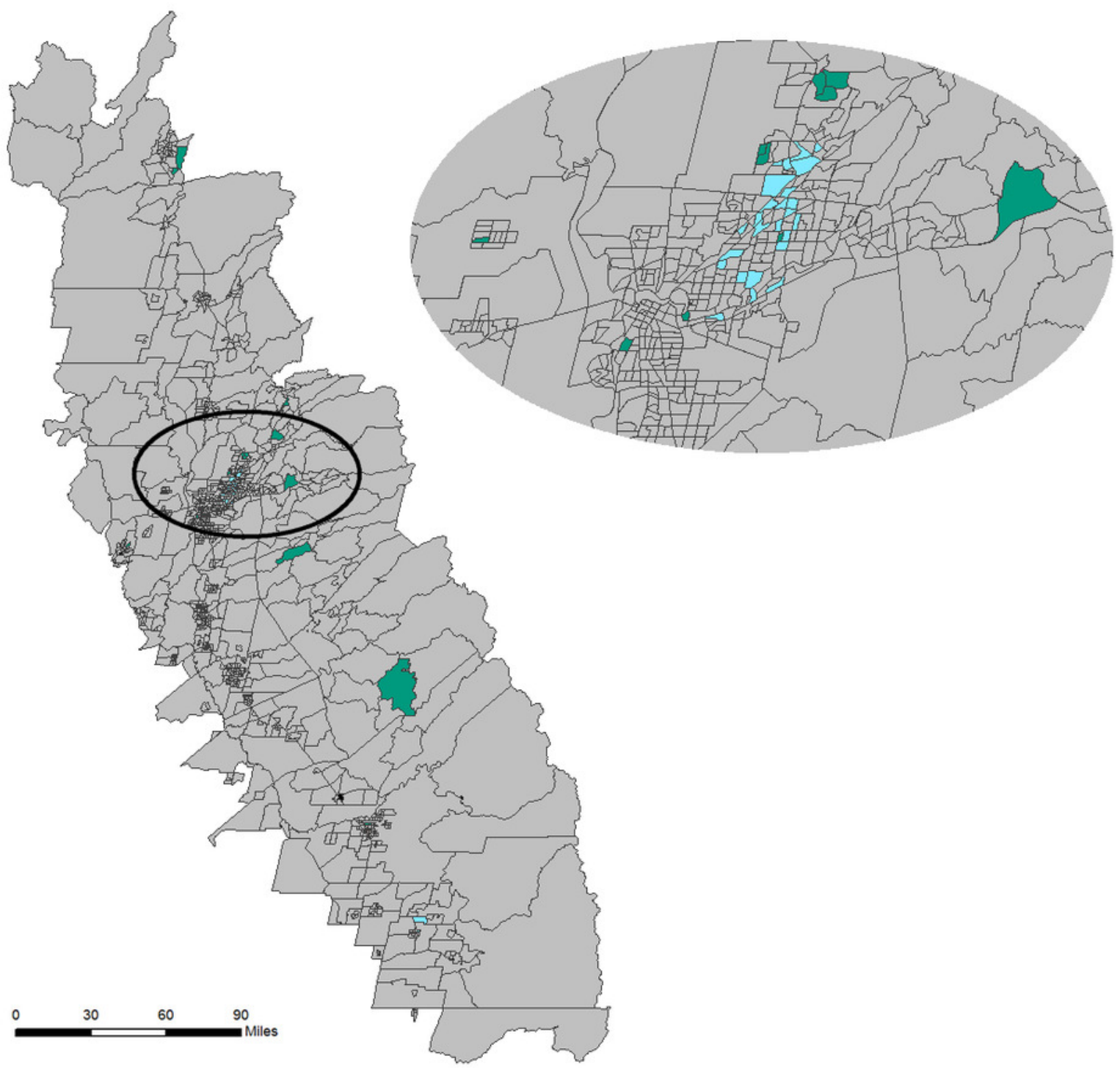


Figure 9

Parallel Coordinate Plot (PCP) highlighting the 5 clusters found in Glenn County, the county with the highest human incidence rate of West Nile Virus (WNV).

Pink line represents the only cluster that contains more than 1 census tract in Glenn County.

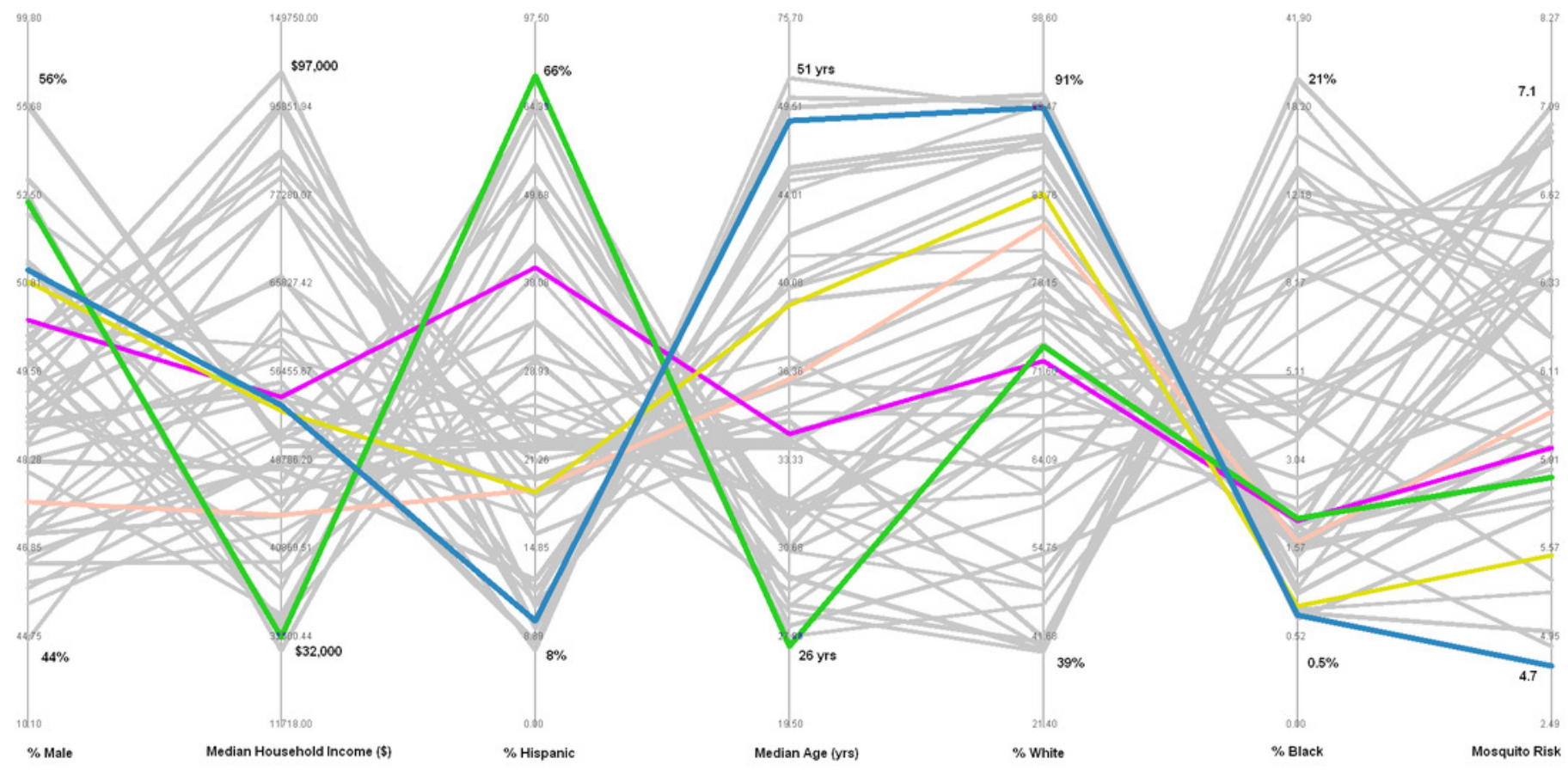




\section{Figure 10}

Census tracts, highlighted in pink, within 1-standard deviation ellipse that are in the same cluster that contains more than one census tract found in Glenn County.

Built using Topologically Integrated Geographic Encoding and Referencing system (TIGER) basemap files. Sources for basemap: U.S. Census Bureau.
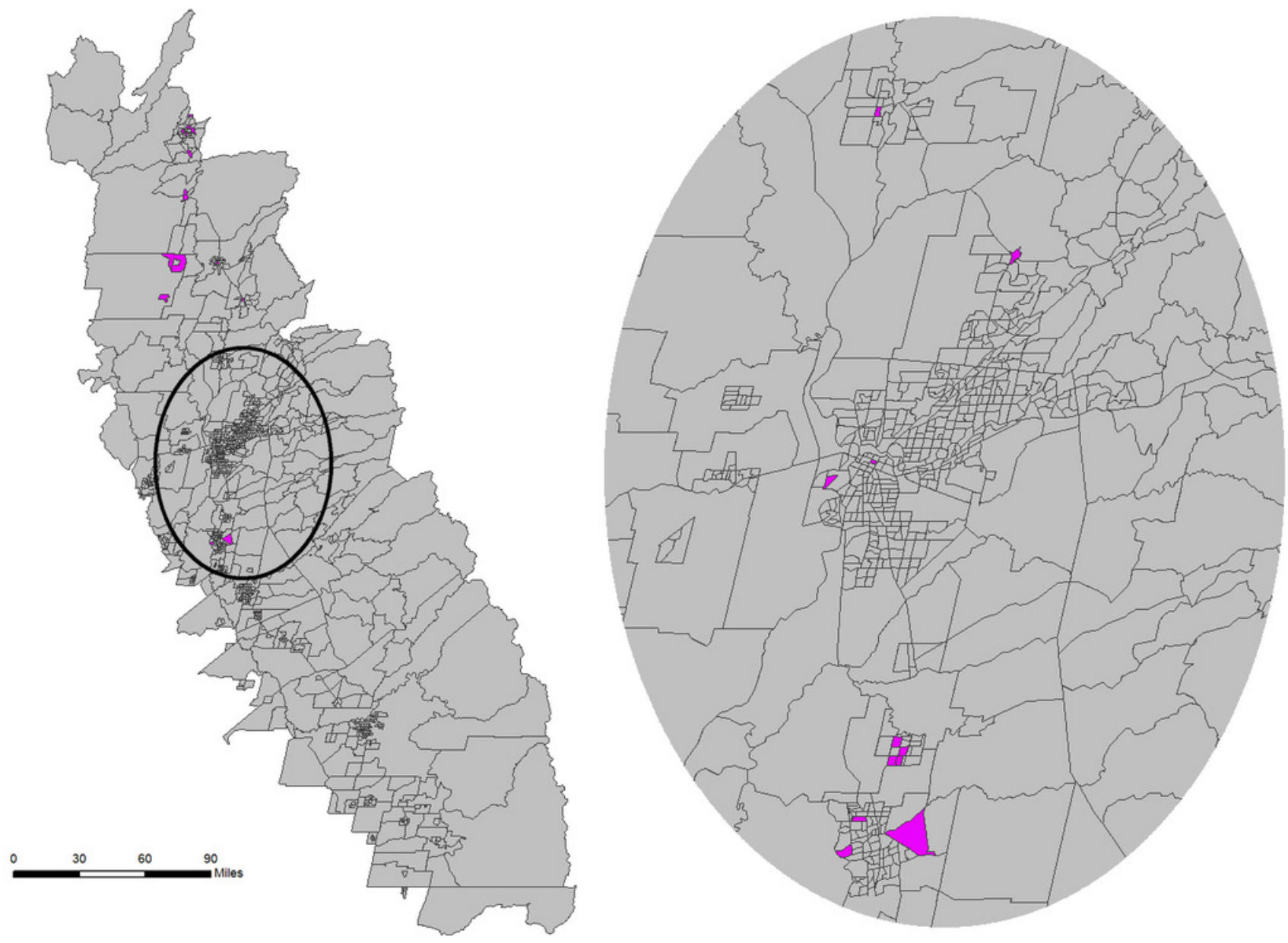